\title{
An Assay to Determine Mechanisms of Rapid Autoantibody-Induced Neurotransmitter Receptor Endocytosis and Vesicular Trafficking in Autoimmune Encephalitis
}

OPEN ACCESS

Edited by:

Stefan Bittner,

Johannes Gutenberg University

Mainz, Germany

Reviewed by:

Chiara Cordiglieri,

Istituto Nazionale Genetica Molecolare

(INGM), Italy

Christoph Kleinschnitz,

Universität Würzburg, Germany

Erhard Wischmeyer,

Universität Würzburg, Germany

${ }^{*}$ Correspondence:

Nico Melzer

nico.melzer@ukmuenster.de

Specialty section:

This article was submitted to

Multiple Sclerosis and

Neuroimmunology,

a section of the journal

Frontiers in Neurology

Received: 19 September 2018 Accepted: 11 February 2019

Published: 01 March 2019

Citation:

Amedonu E, Brenker C, Barman S, Schreiber JA, Becker S, Peischard S, Strutz-Seebohm N, Strippel C, Dik A,

Hartung $H-P$, Budde $T$, Wiendl $H$,

Strünker T, Wünsch B, Goebels N,

Meuth SG, Seebohm G and Melzer N

(2019) An Assay to Determine Mechanisms of Rapid

Autoantibody-Induced

Neurotransmitter Receptor Endocytosis and Vesicular Trafficking

in Autoimmune Encephalitis.

Front. Neurol. 10:178.

doi: 10.3389/fneur.2019.00178
Elsie Amedonu ${ }^{1,2}$, Christoph Brenker ${ }^{3}$, Sumanta Barman ${ }^{4}$, Julian A. Schreiber ${ }^{1}$, Sebastian Becker ${ }^{1}$, Stefan Peischard ${ }^{1}$, Nathalie Strutz-Seebohm ${ }^{1}$, Christine Strippel ${ }^{2}$, Andre Dik ${ }^{2}$, Hans-Peter Hartung ${ }^{4}$, Thomas Budde ${ }^{5}$, Heinz WiendI ${ }^{2}$, Timo Strünker ${ }^{3}$, Bernhard Wünsch ${ }^{6}$, Norbert Goebels ${ }^{4}$, Sven G. Meuth ${ }^{2}$, Guiscard Seebohm ${ }^{1}$ and Nico Melzer ${ }^{\text {* }}$

${ }^{1}$ Myocellular Electrophysiology and Molecular Biology, Institute for Genetics of Heart Diseases, University of Muenster, Muenster, Germany, ${ }^{2}$ Department of Neurology, University of Muenster, Muenster, Germany, ${ }^{3}$ Centre of Reproductive Medicine and Andrology, University of Muenster, Muenster, Germany, ${ }^{4}$ Department of Neurology, Universitätsklinikum and Center for Neurology and Neuropsychiatry LVR Klinikum, Heinrich Heine University Duesseldorf, Duesseldorf, Germany, ${ }^{5}$ Institute for Physiology I, University of Muenster, Muenster, Germany, ${ }^{6}$ Institute for Pharmaceutical and Medical Chemistry, University of Muenster, Muenster, Germany

N-Methyl-D-aspartate (NMDA) receptors (NMDARs) are among the most important excitatory neurotransmitter receptors in the human brain. Autoantibodies to the human NMDAR cause the most frequent form of autoimmune encephalitis involving autoantibody-mediated receptor cross-linking and subsequent internalization of the antibody-receptor complex. This has been deemed to represent the predominant antibody effector mechanism depleting the NMDAR from the synaptic and extra-synaptic neuronal cell membrane. To assess in detail the molecular mechanisms of autoantibody-induced NMDAR endocytosis, vesicular trafficking, and exocytosis we transiently co-expressed rat GluN1-1a-EGFP and GluN2B-ECFP alone or together with scaffolding postsynaptic density protein 95 (PSD-95), wild-type (WT), or dominant-negative (DN) mutant Ras-related in brain (RAB) proteins (RAB5WT, RAB5DN, RAB11WT, RAB11DN) in HEK 293T cells. The cells were incubated with a pH-rhodamine-labeled human recombinant monoclonal GluN1 IgG1 autoantibody (GluN1-aAb ${ }^{\text {pH-rhod) }}$ genetically engineered from clonally expanded intrathecal plasma cells from a patient with anti-NMDAR encephalitis, and the $\mathrm{pH}$-rhodamine fluorescence was tracked over time. We show that due to the acidic luminal $\mathrm{pH}$, internalization of the NMDAR-autoantibody complex into endosomes and lysosomes increases the $\mathrm{pH}$-rhodamine fluorescence. The increase in fluorescence allows for mechanistic assessment of endocytosis, vesicular trafficking in these vesicular compartments, and exocytosis of the NMDAR-autoantibody complex under steady state conditions. Using this method, we demonstrate a role for PSD-95 in stabilization of NMDARs in the cell membrane in the presence of GluN1-aAbpH-rhod, while RAB proteins did not exert a significant effect on vertical trafficking of 
the internalized NMDAR autoantibody complex in this heterologous expression system. This novel assay allows to unravel molecular mechanisms of autoantibody-induced receptor internalization and to study novel small-scale specific molecular-based therapies for autoimmune encephalitis syndromes.

Keywords: autoimmune encephalitis, N-Methyl-D-aspartate receptors, cross-linking, endocytosis, vesicular trafficking, exocytosis, autoantibodies

\section{INTRODUCTION}

Most of the glutamatergic signaling mechanisms in the central nervous system (CNS) rely on the binding of this neurotransmitter (NT) to specific glutamate receptors (GluRs). Ionotropic ligand-gated ion channels (iGluRs) and metabotropic G protein-coupled receptors (mGluRs) mediate fast and slow glutamatergic excitatory synaptic transmission at synapses between neuronal axons and dendrites (1). The iGluRs include the slow, modulatory N-Methyl-D-aspartate receptors (NMDARs), the fast $\alpha$-Amino-3-hydroxy-5-methyl4 -isoxazolepropionic acid receptors (AMPARs) (2), and the Kainate receptors (KARs), which typically do not contribute to baseline synaptic transmission.

Functional adult neuronal NMDARs are hetero-tetrameric complexes, formed predominantly by two GluN1 and two GluN2 subunits (3). The subunits share a similar membrane topology, i.e., four transmembrane domains (M1-M4), a reentrant membrane loop between M3 and M4 domains, and long extracellular $\mathrm{N}$ - and intracellular C-termini (relatively short for GluN1) (4). Hallmarks of NMDARs include voltagesensitive block by extracellular $\mathrm{Mg}^{2+}$, slow current kinetics, and high $\mathrm{Ca}^{2+}$ permeability (1). Thereby, NMDARs serve a crucial function in synaptic plasticity (expressed as a change in receptor number and functional properties), learning, and memory. These processes start with the release of glutamate from presynaptic axon terminals and the subsequent binding together with the coagonist glycine mainly to postsynaptic NMDARs. Postsynaptic NMDARs, in turn, are associated with and regulated by several proteins that together constitute the postsynaptic density (PSD), an elaborate complex of interlinked proteins and elements of the cytoskeleton.

Neuronal glutamate receptor trafficking is a multi-step process that involves protein synthesis at the dendritic tree of the postsynaptic neuron, receptor subunit quality control and

\footnotetext{
Abbreviations: AMPA, $\alpha$-Amino-3-hydroxy-5-methyl-4-isoxazolepropionic acid; AMPAR, $\alpha$-Amino-3-hydroxy-5-methyl-4-isoxazolepropionic acid receptor; CNS, central nervous system; DMEM, Dulbecco's modified Eagle's medium; DN, dominant-negative; ECFP, enhanced cyan fluorescent protein; EE, early endosomes; EGFP, enhanced green fluorescent protein; ER, endoplasmic reticulum; FBS, fetal bovine serum; GluN1-aAb $\mathrm{p}^{\mathrm{pH}-\mathrm{rhod}}, \mathrm{pH}$-rhodamine-labeled human recombinant monoclonal GluN1 IgG1 autoantibody; GA, Golgi apparatus; GC, Golgi complex; GluR, glutamate receptors; Ig, immunoglobulin; iGluR, ionotropic glutamate receptor; KAR, kainate receptor; NEAA, non-essential amino acids; NMDA, N-Methyl-D-aspartate; NMDAR, N-Methyl-D-aspartate receptor; NT, neurotransmitter; mEPSCs, miniature excitatory post-synaptic currents; mGluR, metabotropic G-protein coupled receptor; PSD-95, postsynaptic density protein 95; RAB, Ras-related in brain; RE, recycling endosomes; RV, recycling vesicle; WT, wild-type.
}

assemblage in the endoplasmic reticulum (ER), processing in the Golgi apparatus (GA), vesicular packaging in the Golgi complex (GC), subsequent vertical trafficking to the neuronal cell surface membrane and anchorage at the PSD, lateral trafficking into and out of the PSD, as well as the internalization (endocytosis), subsequent neuronal surface membrane reinsertion (exocytosis) carried out by endosomes (vertical trafficking) or degradation carried out by lysosomes (5). At each step of the trafficking process, NMDARs associate with specific partner proteins that allow for their maturation and/or transportation (4).

Glutamate receptors are major targets in autoimmune encephalitis syndromes $(6,7)$, in which autoantibodies of the immunoglobulin (Ig) G type target iGluRs like NMDARs (8) and AMPARs (9) as well as mGluRs like metabotropic glutamate receptor 1 (mGluR1) (10) and 5 (mGluR5) (11). These autoantibodies disrupt receptor function, cross-link receptors leading to internalization of the antibody-receptor complex (9, 12-15), and activate complement depending on the autoantibody, its IgG subclass, and the complement concentration in the cerebrospinal fluid.

NMDAR autoantibodies are of the IgG 1 or 3 subtypes and can directly affect the gating of the receptor (16). Residues $\mathrm{N}^{368} / \mathrm{G}^{369}$ in the extracellular domain of the GluN1 subunit of NMDARs may form part of the immunodominant binding region for IgG on the receptor molecule. In singlechannel recordings, antibody binding to the receptor instantly caused more frequent openings and prolonged open times of the receptor (16). Moreover, NMDAR autoantibodies caused selective and reversible decrease in postsynaptic surface density and synaptic anchoring of NMDAR in both glutamatergic and GABAergic rat hippocampal neurons by disrupting the interaction of NMDAR with Ephrin-B2 receptors (17), followed by selective NMDAR cross-linking and internalization $(13,14)$. Consistently, NMDAR antibodies selectively decreased NMDARmediated miniature excitatory post-synaptic currents (mEPSCs) without affecting AMPAR-mediated mEPSCs in cultured rat hippocampal neurons (13).

In cultured rat hippocampal neurons, once internalized, antibody-bound NMDAR traffic through recycling endosomes and lysosomes, but do not induce compensatory changes in glutamate receptor gene expression (14). The internalized antibody-receptor complexes co-localize rather with RAB11positive recycling endosomes than with Lamp1-positive lysosomes suggesting subsequent recycling and exocytosis (14). The process of NMDAR internalization plateaus after $12 \mathrm{~h}$, reaching a steady state that persists throughout the duration of the antibody treatment (14), likely reflecting a state of equilibrium between the rate of receptor internalization and the 
rate of receptor (re-)insertion from different compartments into the surface membrane (14).

Probably due to the lack of blood-brain barrier disruption in NMDAR encephalitis and subsequent lack of relevant complement concentrations in the cerebrospinal fluid, as well as internalization of NMDAR together with the autoantibodies, no complement depositions or major neuronal loss could be detected in biopsy specimens of patients with NMDAR encephalitis, despite large numbers of intracerebral autoantibody-secreting plasma cells $(18,19)$. Indeed, fully reversible impairment of behavior and memory occurs in mice receiving passive intrathecal transfer of NMDAR autoantibodies $(20,21)$ that is prevented by co-application of ephrin (22).

The effects on receptor-mediated currents are rather small in heterologous expression systems and do not allow for mechanistic studies on autoantibody-induced neurotransmitter receptor internalization and trafficking in anti-NMDAR encephalitis and other forms of autoimmune encephalitis (21). Thus, the aim of this study was to develop an assay suitable to study in molecular detail the mechanism of autoantibody-induced NMDAR endocytosis, vesicular trafficking, and exocytosis and potentially to study novel small-scale specific molecular-based therapies for autoimmune encephalitis syndromes.

\section{MATERIALS AND METHODS}

\section{Construction of NMDAR Expression Vectors}

NMDAR constructs were kindly provided by Prof. Michael Hollmann, Receptor Biochemistry, Faculty of Chemistry and Biochemistry, Ruhr University Bochum, Germany. cDNAs encoding the rat GluN1-1a and GluN2B NMDAR subunits were sub-cloned into the pEGFP-N1 and pECFP-N1 mammalian expression vectors, respectively. To allow for the visualization of the subunits, enhanced cyan fluorescent protein (ECFP), and enhanced green fluorescent protein (EGFP) were inserted in-frame at the $\mathrm{N}$-terminus of the subunits. The subunitcontaining plasmids were amplified via growth in $E$. coli followed by purification based on a modified alkaline lysis procedure (QIAGEN Miniprep kit).

The generation of PSD-95 as well as WT and DN RAB5 and RAB11 expression vector constructs has been described elsewhere $(5,23)$.

\section{HEK 293T Cell Co-transfection}

HEK 293T cells were cultured in growth media comprising high-glucose Dulbecco's modified Eagle's medium (DMEM) supplemented with $10 \%$ fetal bovine serum (FBS), non-essential amino acids (NEAA), Pen-Strep, and $2 \mathrm{mM}$ glutamine. Two days prior to transfection, exponentially growing cells were seeded on poly-D-lysine-coated glass bottom 96 -well-plates to a density of approximately $5.0-8.0 \times 10^{5}$ /well. Two hours prior to co-transfection, the culture medium was replaced with fresh culture medium. HEK 293T cells were then transiently cotransfected with the cDNAs $(0.25 \mu \mathrm{g}$ GluN1-1a-EGFP and 0.25 $\mu \mathrm{g}$ GluN2B-ECFP) encoding the NMDAR subunits as well as
PSD-95, or WT, or DN RAB proteins using the FuGene HD (Promega Corporations) transfection technique according to manufacturer's instructions or left untransfected. $24 \mathrm{~h}$ post cotransfection, cells were seeded onto poly-D-lysine-coated glass bottom 96-well-plates. Confocal laser-scanning microscopy was used to quantify cell-surface NMDAR density ( $\times 63$ glycerol objective; TCS-SP5 Leica- Microsystems, Germany).

\section{pH-rhodamine Labeling of a Human Recombinant Monoclonal GluN1 Autoantibody}

Generation of a recombinant human monoclonal GluN1 autoantibody (GluN1-aAb) engineered from clonally expanded intrathecal plasma cells of a patient with anti-NMDAR encephalitis has recently been described (21). Labeling of GluN1$\mathrm{aAb}$ was performed using a $\mathrm{pHrodo}^{\mathrm{TM}}$ Red Microscale Labeling Kit (Thermofisher Scientific) according to the recommendations of the supplier. Briefly, $100 \mu \mathrm{L}$ of GluN1-aAb solution $(1 \mathrm{mg} / \mathrm{mL}$ in PBS) were transferred to a "component D" containing reaction tube and supplemented with $10 \mu \mathrm{L}$ of $1 \mathrm{M}$ sodium bicarbonate. pHrodo red succinimidyl ester was dissolved in $10 \mu \mathrm{L}$ of DMSO. From the resulting solution, $0.70 \mu \mathrm{L}$ (as calculated according to the equation 1 of the labeling kit's protocol) was added to the reaction tube containing the $\mathrm{pH}$-adjusted GluN1-aAb. This reaction mixture was incubated for $15 \mathrm{~min}$ at RT to allow conjugation. For separation from unbound dye, the reaction mix was spun through a resin-containing column (provided with the kit) at $1,000 \mathrm{~g}$ for $5 \mathrm{~min}$. The purified $\mathrm{pH}$-rhodo red labeled GluN1-aAb (GluN1-aAb ${ }^{\mathrm{pH}-\text { rhod }}$ ) was recovered from the collection tube and stored aliquoted at $-20^{\circ} \mathrm{C}$, while unbound dye remained in the resin.

\section{Co-incubation of GluN1-aAb ${ }^{\text {pH-rhod }}$ With HEK 293T Cells Expressing GluN1-1a-EGFP/GluN2B-ECFP NMDARs and Fluorescence Intensity Analysis}

The rhodamine fluorophore possesses a well-known $\mathrm{pH}$ - and temperature-dependent fluorescence quantum yield (24), which decreases linearly as $\mathrm{pH}$ and temperature increases (25). These physicochemical properties needed to be considered in our experimental setting.

Untransfected or co-transfected HEK 293T cells from the same 96-well-plate were incubated with GluN1-aAb ${ }^{\mathrm{pH}-\text { rhod }}$ at a concentration of $4 \mu \mathrm{g} / \mathrm{ml}$ in phosphate-buffered saline (PBS, $\mathrm{pH}$ 7.4) for $2 \mathrm{~min}$. After that, unbound GluN1-aAb $\mathrm{p}^{\mathrm{pH}-\text { rhod }}$ was washed-out by superfusing 96-wells with PBS to yield cellbound GluN1-aAb $\mathrm{p}^{\mathrm{H}-\text { rhod }}$ fluorescence. Wells on the same 96well-plate without cells incubated with GluN1-aAb $b^{\mathrm{H}-\text { rhod }}$ at a concentration of $4 \mu \mathrm{g} / \mathrm{ml}$ in PBS without wash-off served as control. All incubations were conducted at $4^{\circ} \mathrm{C}$ on ice to prevent endocytosis prior to recordings.

Subsequently, fluorescence of GluN1-EGFP and GluN1$\mathrm{aAb}^{\mathrm{pH}-\text { rhod }}$ was excited at 480 and $520 \mathrm{~nm}$ and detected at $510 \mathrm{~nm}$ (confocal imaging) and $580 \mathrm{~nm}$ (plate reader), respectively, verifying GluN1-1a-EGFP expression and 
presence of the GluN1-aAb-bound rhodamine fluorescence (GluN1-aAb ${ }^{\mathrm{pH}-\mathrm{rhod}}$ ).

Subsequently, the temperature was increased rapidly from 4 to $30^{\circ} \mathrm{C}$ to allow for endocytosis, and $\mathrm{pH}$-rhodaminefluorescence was repetitively excited at $520 \mathrm{~nm}$ and the emission was detected at $580 \mathrm{~nm}$. The overall $\mathrm{pH}$-rhodamine fluorescence decreased exponentially with time reaching a steady state after approximately $500 \mathrm{~s}$ mainly reflecting the known temperaturedependent fluorescence quantum yield of rhodamine under all experimental conditions.

The steady state $\mathrm{pH}$-rhodamine-fluorescence intensity at $580 \mathrm{~nm}$ after $500 \mathrm{~s}$ of HEK 293T cells expressing GluN11a-EGFP/GluN2B-ECFP NMDARs was significantly higher compared to untransfected HEK 293T cells and served as a cumulative measure of endocytosis of GluN1-aAb $\mathrm{pH}^{\mathrm{H}-\mathrm{rhod}}$ bound to the NMDAR with subsequent acidification within endosomes and/or lysosomes and exocytosis. This allowed for mechanistic studies in HEK 293T cells expressing GluN1-1aEGFP/GluN2B-ECFP NMDARs co-transfected with scaffolding protein PSD-95 as well as RAB5WT and RAB5DN (mediating vesicle endocytosis) and RAB11WT or RAB11DN (mediating vesicle exocytosis).

\section{Statistical Analysis}

Data was analyzed using Origin 9 (OriginLab Corporation). Oneway ANOVA followed by multiple pair-wise comparisons with Bonferroni's post-hoc correction was used to statistically analyze differences in fluorescence intensity; $p \leq 0.05$ were considered as significant; data in figures were expressed as mean \pm SEM. All experiments were performed in triplicates.

\section{RESULTS}

To assess in detail the molecular mechanisms of NMDAR autoantibody-induced NMDAR endocytosis, vesicular trafficking, and exocytosis we transiently expressed rat GluN11a-EGFP and GluN2B-ECFP alone or together with PSD-95 or with WT- or DN-mutant RAB proteins (RAB5WT, RAB5DN, RAB11WT, RAB11DN) in HEK 293T cells. As a control, HEK 293T cells were left untransfected.

The cells were incubated with a pH-rhodamine-labeled human recombinant monoclonal GluN1 IgG1 autoantibody [GluN1-aAb $\left.{ }^{\mathrm{pH}-\text { rhod }},(21)\right]$.

We surmised that the $\mathrm{pH}$-rhodamine fluorescence is increased during the ensuing internalization of the NMDAR-autoantibody complex, due to the acidic luminal $\mathrm{pH}$ of endosomes and lysosomes. This might allow for mechanistic assessment of endocytosis, vesicular trafficking in both vesicular compartments and exocytosis of the NMDAR-autoantibody complex (for assay design see Figure 1).

In a first set of experiments, HEK $293 \mathrm{~T}$ cells were transiently co-transfected only with rat GluN1-1a-EGFP and GluN2B-ECFP or left untransfected. After 2 days, expression of fluorescently labeled NMDARs was verified using confocal laser-scanning microscopy. About $70-80 \%$ of the cells expressed GluN1-1aEGFP as subunit putatively targeted by the GluN1-aAb ${ }^{\mathrm{pH}-\text { rhod }}$ (Figure 2A) and GluN2B-ECFP (data not shown). Longer

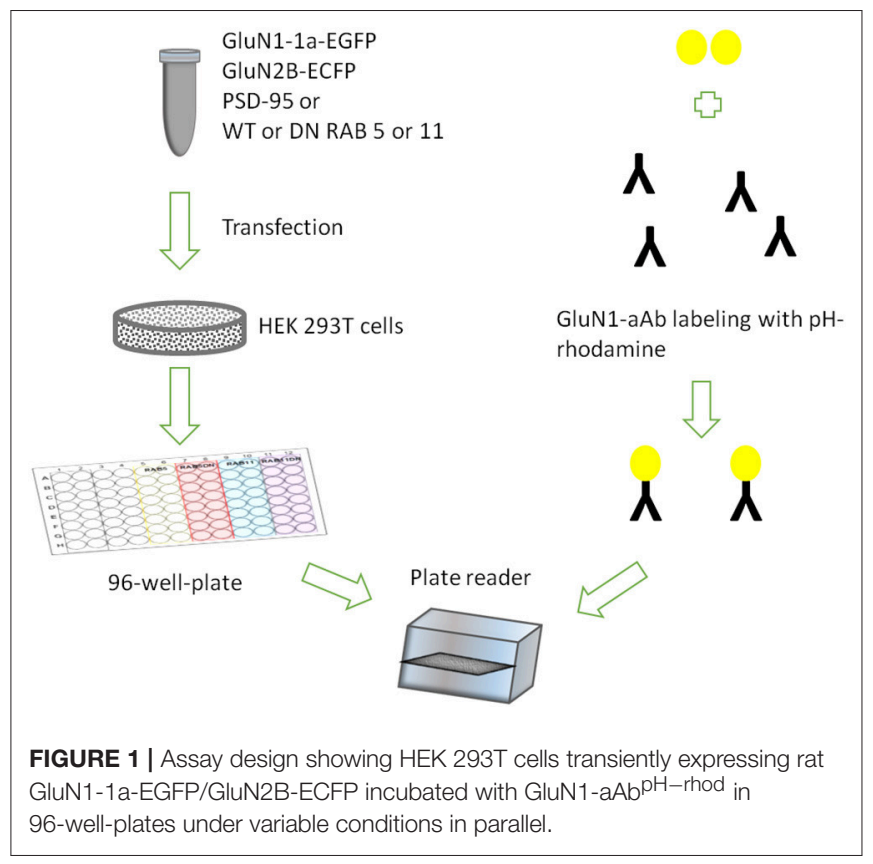

expression times or higher amounts of NMDAR-cDNA for transfections decreased expression levels, supposedly due to cytotoxic effects of pronounced overexpression of NMDARs.

Next, GluN1-1a-EGFP- and GluN2B-ECFP-transfected and untransfected cultured cells were incubated with GluN1$\mathrm{aAb}^{\mathrm{pH}-\mathrm{rhod}}$. The incubation was performed at $4^{\circ} \mathrm{C}$ to stop ongoing endocytosis. After that, unbound GluN1-aAb $\mathrm{b}^{\mathrm{pH}-\text { rhod }}$ was washed-off. As a control, wells without cells were incubated with GluN1-aAb ${ }^{\mathrm{pH}-\text { rhod }}$ without wash-off.

Subsequently, GluN1-1a-EGFP and GluN1-aAb ${ }^{\mathrm{pH}-\text { rhod }}$ fluorescence was excited at $480 \mathrm{~nm}$ and $520 \mathrm{~nm}$ and measured at 510 and $580 \mathrm{~nm}$, respectively, verifying GluN1-1a-EGFP expression of transfected but not untransfected cells and presence of the GluN1-aAb-bound rhodamine fluorescence (GluN1-aAb ${ }^{\mathrm{pH}-\text { rhod }}$, Figure 2B).

The plate was subsequently transferred to a fluorescence plate reader for time-resolved detection of the $\mathrm{pH}$-rhodamine fluorescence intensity at $30^{\circ} \mathrm{C}$. The temperature was increased rapidly from $4-30^{\circ} \mathrm{C}$ to start endocytosis, and $\mathrm{pH}$-rhodaminefluorescence was repetitively excited at $520 \mathrm{~nm}$ and the emission was detected at $580 \mathrm{~nm}$. The overall rhodamine fluorescence at $580 \mathrm{~nm}$ decreased exponentially with time, reaching a steady state after approximately $500 \mathrm{~s}$ (Figure 3B) mainly reflecting the known temperature-dependent fluorescence quantum yield of rhodamine (24-26) under all experimental conditions.

Of note, the fluorescence-spectrum did not change over time (Figure 3A), illustrating that $\mathrm{pH}$-rhodamine fluorescence was detected throughout the experiments. Moreover, steadystate fluorescence intensities of empty wells without wash-off of GluN1-aAb $b^{\mathrm{pH}-\text { rhod }}$ were much larger than those of wells with HEK 293T cells illustrating the known background fluorescence of $\mathrm{pH}$ rhodamine at neutral $\mathrm{pH}$ of 7.4 in PBS (roughly $1 / 3$ of the maximal fluorescence at acidic $\mathrm{pH}$ of 4.0 ) and thus the necessity of the washing step (Figure 3B). 

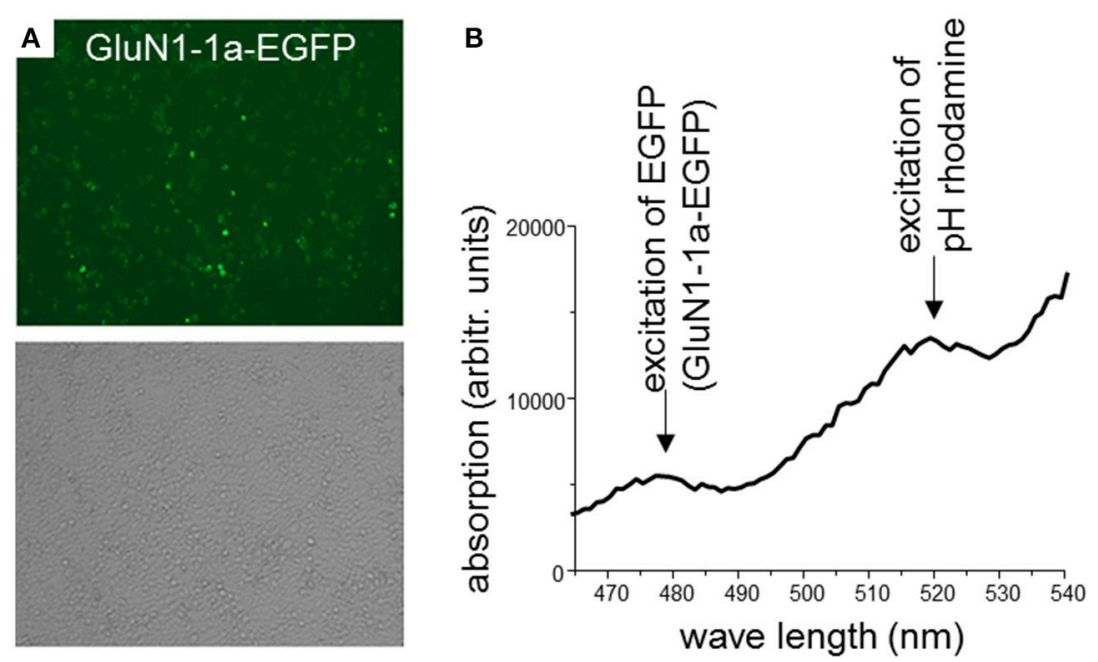

FIGURE 2 | (A) HEK 293T cells co-expressing rat GluN1-1a-EGFP and GluN2B-ECFP. 2 days after transfection, expression of fluorescently labeled NMDARs was verified using confocal laser-scanning microscopy. About 70-80\% of the cells expressed GluN1-1a-EGFP as subunit putatively targeted by the GluN1-aAbPH-rhod (upper panel) and GluN2B-ECFP (data not shown). Light microscopy demonstrates typical cell densities (lower panel). (B) Binding of GluN1-aAbpH-rhod to the NMDAR subunits as determined by the plate reader experiment. HEK 293T cells expressing GluN1-1a-EGFP/GluN2B-ECFP were incubated with GluN1-aAb ${ }^{\text {pH-rhod }}$ on ice in 96-well-plates. GluN1-1a-EGFP and GluN1-aAbpH-rhod fluorescence was excited at wavelengths of $480 \mathrm{~nm}$ and $520 \mathrm{~nm}$ and emission measured at wavelengths of 510 and $580 \mathrm{~nm}$, respectively, verifying the GluN1-1a-EGFP expression and the binding of GluN1-aAb $\mathrm{b}^{\mathrm{pH}-\mathrm{rhod}}$.
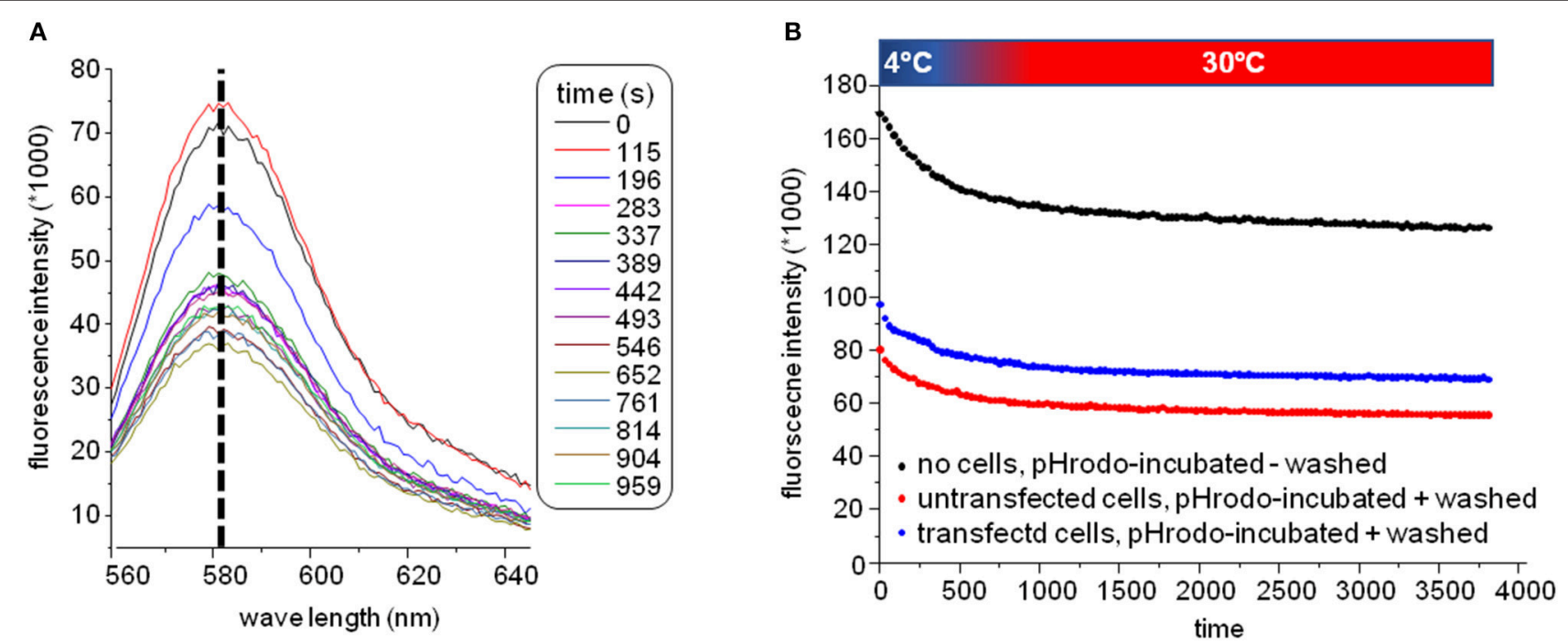

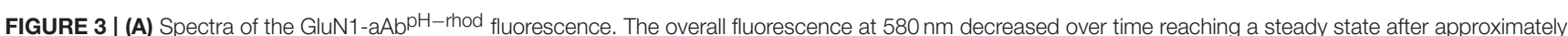
500 s mainly reflecting the known temperature-dependent fluorescence quantum yield of rhodamine (24-26) elicited by elevating the temperature for $4-30^{\circ} \mathrm{C}$ at the beginning of the experiment under all experimental conditions. During this decay, the excitation spectrum did not shift/change indicating that indeed pH-rhodamine-fluorescence was detected throughout the whole experiment. (B) Representative time-dependent traces of the GluN1-aAb ${ }^{\mathrm{pH}-\text { rhod }}$ fluorescence

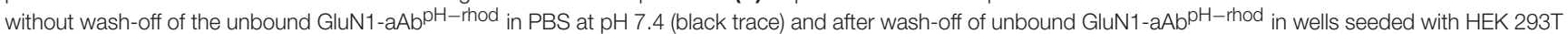
cells expressing GluN1-1a-EGFP/GluN2B-ECFP (blue trace) or untransfected HEK 293T cells (red trace). The steady state pH-rhodamine-fluorescence intensity at $580 \mathrm{~nm}$ after $500 \mathrm{~s}$ of HEK 293T cells expressing GluN1-1a-EGFP/GluN2B-ECFP NMDARs was significantly higher compared to the background fluorescence of untransfected HEK 293T cells and served as a cumulative measure of endocytosis of GluN1-aAb ${ }^{\text {pH-rhod }}$ bound to the NMDAR with subsequent acidification within endosomes and/or lysosomes and exocytosis.

The steady state $\mathrm{pH}$-rhodamine-fluorescence intensity at $580 \mathrm{~nm}$ after $500 \mathrm{~s}$ of HEK 293T cells expressing GluN11a-EGFP/GluN2B-ECFP NMDARs was significantly higher compared to the background fluorescence of untransfected HEK 293T cells (Figure 4) and served as a cumulative measure of endocytosis of GluN1-aAb ${ }^{\mathrm{pH}-\text { rhod }}$ bound to the NMDAR with 


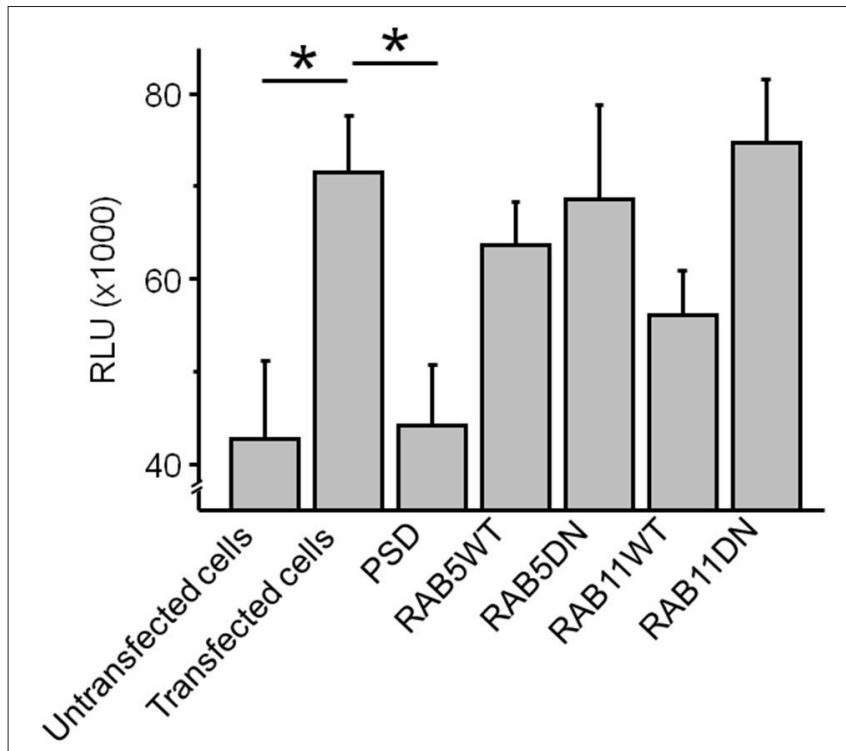

FIGURE 4 | The co-expression of GluN1-1a-EGFP/GluN2B-ECFP NMDARs with the scaffolding protein PSD-95 significantly reduced the cumulative steady state $\mathrm{pH}$-rhodamine-fluorescence intensity at $580 \mathrm{~nm}$ after $500 \mathrm{~s}$ toward background levels of untransfected HEK 293T cells. In contrast, co-transfection with RAB5WT or RAB5DN (mediating/inhibiting endocytosis) or RAB11 WT or RABDN (mediating/inhibiting exocytosis) did not significantly affect the cumulative steady state $\mathrm{pH}$-rhodamine-fluorescence intensity at $580 \mathrm{~nm}$ after $500 \mathrm{~s}$ in this heterologous expression system. One-way ANOVA followed by multiple pair-wise comparisons with Bonferroni's post-hoc correction was used to statistically analyze differences in fluorescence intensity; $p \leq 0.05$ were considered as significant ( $\left(^{\star}\right)$; data are expressed as mean \pm SEM. All experiments were performed in triplicates.

subsequent acidification within endosomes and/or lysosomes and exocytosis. This allowed for mechanistic studies in HEK 293T cells expressing GluN1-1a-EGFP/GluN2B-ECFP NMDARs co-transfected with scaffolding protein PSD-95 as well as WT and DN RAB5 (mediating vesicle endocytosis) and RAB11 (mediating vesicle exocytosis).

The co-expression of GluN1-1a-EGFP/GluN2B-ECFP NMDARs with the scaffolding protein PSD-95 significantly reduced the cumulative steady state $\mathrm{pH}$-rhodamine-fluorescence intensity at $580 \mathrm{~nm}$ after $500 \mathrm{~s}$ toward background levels of untransfected HEK 293T cells (Figure 4). In contrast, cotransfection with RAB5WT or RAB5DN (mediating/inhibiting endocytosis) or RAB11 WT or RABDN (mediating/inhibiting exocytosis) did not significantly affect the cumulative steady state $\mathrm{pH}$-rhodamine-fluorescence intensity at $580 \mathrm{~nm}$ after $500 \mathrm{~s}$ in this heterologous expression system (Figure 4).

\section{DISCUSSION}

NMDARs are among the most important excitatory receptors in the human brain. NMDAR autoantibodies cause encephalitis by binding to NMDARs, transducing conformational changes and subsequent endocytosis $(21,27)$. Recently, we showed that pre-incubation for an hour of a recombinant human monoclonal GluN1 autoantibody engineered from clonally expanded intrathecal plasma cells of a patient with anti-NMDAR encephalitis reduced NMDAR-mediated currents recorded from Xenopus laevis oocytes by about 20\% (21). This result is similar to previous results in Xenopus laevis oocytes, showing a timedependent inhibition of steady-state NMDAR-mediated currents of about $30 \%$ within 16 min upon exposure to dialysed sera of patients with anti-NMDAR encephalitis (28). To record NMDAR-mediated currents in Xenopus laevis oocytes (and other heterologous expression systems), it is required to use $\mathrm{Ca}^{2+}$-free media to block current inactivation (29). This might explain the rather small antibody-mediated action in oocytes (and probably other heterologous expression systems) compared to the pronounced effects on NMDAR expression on neuronal cell surface in vitro, ex vivo, and on memory impairment in vivo in mice. The $\mathrm{Ca}^{2+}$-free recording conditions may cause conformational changes of the NMDAR induced by binding of the antibody or modulate antibody binding itself and thus diminish subsequent receptor cross-linking and internalization.

These effects on receptor-mediated currents in Xenopus laevis oocytes (and other heterologous expression systems) do not allow for further mechanistic studies on autoantibodyinduced neurotransmitter receptor internalization and trafficking in anti-NMDAR encephalitis and other forms of autoimmune encephalitis (30). Thus, the aim of this study was to develop an assay suitable for this kind of study.

We used a $\mathrm{pH}$-rhodamine labeled single recombinant human GluN1 IgG1 autoantibody [GluN1-aAb $\mathrm{p}^{\mathrm{pH}-\mathrm{rhod}}$, (21)]. This monoclonal autoantibody has previously been shown to evoke all effects of natural NMDAR autoantibodies contained in cerebrospinal fluid of patients with anti-NMDAR encephalitis in vitro and in vivo (21).

We tested the effects of GluN1-aAb $\mathrm{pH}^{\mathrm{H}-\text { rhod }}$ incubation on NMDAR endocytosis, trafficking, and exocytosis mechanisms in HEK 293T cells co-transfected with EGFP-tagged GluN1-1a and ECFP-tagged GluN2B subunits alone or together with PSD-95 or WT- or DN-mutant RAB 5 (mediating endocytosis) and 11 (mediating exocytosis) proteins.

Endocytosis, intracellular trafficking, and exocytosis of the antibody-receptor complex is mediated by transporting vesicles with acidic luminal $\mathrm{pH}$. Thus, we took advantage of this fact, as we found that the use of the steady state GluN1$\mathrm{aAb}^{\mathrm{pH}-\text { rhod }}$ fluorescence in HEK $293 \mathrm{~T}$ cells expressing GluN11a-EGFP/GluN2B-ECFP NMDARs was significantly higher compared to the background fluorescence of untransfected HEK 293 T cells. Thus, this steady state fluorescence served as a cumulative measure of endocytosis of GluN1-aAb $\mathrm{pH}^{\mathrm{H}-\mathrm{rhod}}$ bound to the NMDAR with subsequent acidification within endosomes and/or lysosomes and exocytosis.

Using this approach we could demonstrate a role for PSD-95 for stabilization of NMDAR in the cell membrane in the presence of NMDAR autoantibodies. This suggests that autoantibodyinduced depletion from the cell membrane predominantly affects extra-synaptic NMDARs not associated with PSD-95 and to a lesser extent synaptic NMDARs. This finding is consistent with the notion that autoantibodies through dissociation from clustering ephrinB2 receptors lead to lateral diffusion of synaptic NMDARs within the neuronal cell membrane out to the synapse where they become cross-linked and internalized as extrasynaptic NMDARs $(17,31)$. Cell membrane stabilization of 
synaptic NMDARs [displaying pro-survival functions (32)] and internalization of extra-synaptic NMDARs [displaying cell-death promoting functions (32)] is further consistent with the lack of overt neurodegeneration in NMDAR encephalitis despite excitotoxic excessive extracellular levels of glutamate (33-35).

Endocytosis, intracellular trafficking and exocytosis are under the guidance of small G-proteins of the RAB type. The use of functional WT and DN mutants has been previously successful in identification of intracellular trafficking pathways of glutamate receptors (36). We found that co-transfection with WT or DN RAB5 (mediating/inhibiting endocytosis) did not affect the cumulative steady state GluN1-aAb $b^{\mathrm{HH}-\text { rhod }}$ fluorescence intensity, whereas the cumulative steady state GluN1-aAb $\mathrm{b}^{\mathrm{pH}-\text { rhod }}$ fluorescence intensity was tentatively lowered by co-transfection with WT RAB11 (mediating exocytosis) and tentatively augmented by co-transfection with RAB11DN (inhibiting exocytosis) compared to expression of NMDARs alone. This lack of overt effects of RAB proteins on endocytosis, trafficking and exocytosis of the antibody-receptor complex in our assay is probably due to the overlay by the temperature-dependent fluorescence decrease the amplitude of which is roughly as large as the steady state amplitude of the $\mathrm{pH}$-dependent fluorescence increase upon internalization of the antibody-receptor complex. These opposing effects hinder detailed kinetic analysis of the autoantibody-induced vertical trafficking of the NMDAR performed here.

Hence, given the necessity of washing-off unbound GluN1$\mathrm{aAb}^{\mathrm{pH}-\text { rhod }}$ and halting trafficking during that time by lowering the temperature due to the residual rhodamie fluorescence at $\mathrm{pH} 7.4$ in PBS, the use of fluorophores (that inevitably are also concordantly temperature-sensitive) with an optimized $\mathrm{pH}$-dependence i.e., no fluorescence at physiological $\mathrm{pH}$ of 7.4 might be better suited for our assay. They would enable synchronic adding of the labeled antibody to the cells cultured in $\mathrm{PBS}$ at $\mathrm{pH} 7.4$ at constant temperature of $30^{\circ} \mathrm{C}$ and thus time-resolved tracking of fluorescence increase upon antibodyreceptor internalization.

Taken together, we demonstrate a role for PSD-95 for stabilization of NMDAR in the cell membrane of HEK 293T cells in the presence of NMDAR autoantibodies, while RAB proteins did not exert a significant effect on vertical trafficking of the internalized NMDAR autoantibody complex in this heterologous expression system.

Our assay should be sensitive enough to study novel small-scale specific molecular-based therapies for autoimmune encephalitis that may become feasible as follows:

1) Autoantibody binding to the GluN1 subunit and subsequent induction of conformational changes in the NMDAR could be blocked by antibody fragments, for example. However, with existence of a multitude of autoantibody epitopes within the NMDAR, this approach may not be successful.

2) The autoantibody binding-induced conformational changes within the NMDAR. Small molecule allosteric modulators have recently been developed $(5,37,38)$. Potentially, these compounds may be used to block the conformational changes induced by GluN1-aAb $\mathrm{b}^{\mathrm{pH}-\mathrm{rhod}}$ binding and thus inhibit internalization.
3) Inhibition of the GluN1-aAb $\mathrm{pH}^{\mathrm{H}-\text { rhod }}$-induced internalization. To achieve this, general cellular trafficking pathways have to be blocked. It is questionable if such an approach can be tolerated by patients and would not cause severe side effects.

Taking these considerations into account, development and use of small molecule allosteric modulators may represent a group of drug candidates for anti-NMDAR encephalitis and other forms of autoimmune encephalitis. Evidenced by the relatively robust novel assay, they may be used to screen for compounds that block autoantibody-induced NMDAR cross-linking and internalization. This should always be accompanied by NMDAR stabilization within the synapse to avoid accumulation of NMDAR at extra-synaptic sides of the cell membrane potentially promoting excitotoxic cell death in NMDAR encephalitis.

Therefore, screening results obtained with our assay in HEK 293T overexpressing NMDAR should always be validated using super-resolution microscopy in cultured living neurons and brain slices exhibiting physiological expression levels and subcellular localization of NMDARs.

\section{CONCLUSION}

This novel assay allows to unravel molecular mechanisms of autoantibody-induced receptor internalization and to study novel small-scale specific molecular-based therapies for autoimmune encephalitis syndromes.

\section{DATA AVAILABILITY}

The dataset obtained and analyzed in the current study is available from the corresponding author on a reasonable request.

\section{AUTHOR CONTRIBUTIONS}

$\mathrm{CS}$ and $\mathrm{AD}$ : collected patient samples under the supervision of $\mathrm{HW}, \mathrm{SM}$, and NM; SBa and NG: performed the synthesis and $\mathrm{pH}$-rhodamine labeling of GluN1-aAb $\mathrm{pH}^{\mathrm{H}-\text { rhod }}$; EA, SBe, SP, and JS: performed the transfection, immunocytochemistry, and confocal microscopy with the HEK 293T cells; EA, CB, and TS: together with TB, NS-S, GS, and NM performed incubation of transfected HEK 293T cells with GluN1-aAbpH-rhod and data analysis; H-PH, BW, SM, GS, and NM: designed and supervised the project; EA, SM, GS, and NM: wrote the first draft of the manuscript. All authors contributed to and approved the final version of the manuscript.

\section{FUNDING}

This work was supported by the German Research Foundation (DFG, INST 2105/27-1 to SM), the German Academic Exchange Service (DAAD-MoE postgraduate scholarship to EA), the Walter und Ilse-Rose-Stiftung (to $\mathrm{H}-\mathrm{PH}$ ), the Forschungskommission of the Heinrich-Heine-University Düsseldorf, Germany (to NG) and the Bundesministerium für Bildung und Forschung (BMBF 031A232 to NG). 


\section{ACKNOWLEDGMENTS}

We thank Prof. Michael Hollmann, Receptor Biochemistry, Faculty of Chemistry and Biochemistry, Ruhr University

\section{REFERENCES}

1. Traynelis SF, Wollmuth LP, Mcbain CJ, Menniti FS, Vance KM, Ogden KK, et al. Glutamate receptor ion channels: structure, regulation, and function. Pharmacol Rev. (2010) 62:405-96. doi: 10.1124/pr.109.002451

2. Hollmann M, Heinemann S. Cloned glutamate receptors. Annu Rev Neurosci. (1994) 17:31-108. doi: 10.1146/annurev.ne.17.030194.000335

3. Dingledine R, Borges K, Bowie D, Traynelis SF. The glutamate receptor ion channels. Pharmacol Rev. (1999) 51:7-61.

4. Petralia RS, Al-Hallaq RA, Wenthold RJ. Trafficking and targeting of NMDA receptors. In: Van Dongen AM, editor. Biology of the NMDA Receptor. Boca Raton, FL: CRC Press/Taylor \& Francis (2009).

5. Seebohm G, Neumann S, Theiss C, Novkovic T, Hill EV, Tavare JM, et al. Identification of a novel signaling pathway and its relevance for GluA1 recycling. PLoS ONE. (2012) 7:e33889. doi: 10.1371/journal.pone.0033889

6. Melzer N, Meuth SG, Wiendl H. Paraneoplastic and non-paraneoplastic autoimmunity to neurons in the central nervous system. J Neurol. (2013) 260:1215-33. doi: 10.1007/s00415-012-6657-5

7. Graus F, Titulaer MJ, Balu R, Benseler S, Bien CG, Cellucci T, et al. A clinical approach to diagnosis of autoimmune encephalitis. Lancet Neurol. (2016) 15:391-404. doi: 10.1016/S1474-4422(15)00401-9

8. Dalmau J, Gleichman AJ, Hughes EG, Rossi JE, Peng X, Lai M, et al. Anti-NMDA-receptor encephalitis: case series and analysis of the effects of antibodies. Lancet Neurol. (2008) 7:1091-8. doi: 10.1016/S1474-4422(08)70224-2

9. Lai M, Hughes EG, Peng X, Zhou L, Gleichman AJ, Shu H, et al. AMPA receptor antibodies in limbic encephalitis alter synaptic receptor location. Ann Neurol. (2009) 65:424-34. doi: 10.1002/ana.21589

10. Sillevis Smitt P, Kinoshita A, De Leeuw B, Moll W, Coesmans M, Jaarsma D, et al. Paraneoplastic cerebellar ataxia due to autoantibodies against a glutamate receptor. $N$ Engl J Med. (2000) 342:21-7. doi: 10.1056/NEJM200001063420104

11. Lancaster E, Martinez-Hernandez E, Titulaer MJ, Boulos M, Weaver S, Antoine JC, et al. Antibodies to metabotropic glutamate receptor 5 in the Ophelia syndrome. Neurology. (2011) 77:1698-701. doi: 10.1212/WNL.0b013e3182364a44

12. Coesmans M, Smitt PA, Linden DJ, Shigemoto R, Hirano T, Yamakawa $\mathrm{Y}$, et al. Mechanisms underlying cerebellar motor deficits due to mGluR1autoantibodies. Ann Neurol. (2003) 53:325-36. doi: 10.1002/ana.10451

13. Hughes EG, Peng X, Gleichman AJ, Lai M, Zhou L, Tsou R, et al. Cellular and synaptic mechanisms of anti-NMDA receptor encephalitis. J Neurosci. (2010) 30:5866-75. doi: 10.1523/JNEUROSCI.0167-10.2010

14. Moscato EH, Peng X, Jain A, Parsons TD, Dalmau J, Balice-Gordon RJ. Acute mechanisms underlying antibody effects in anti-N-methyl-D-aspartate receptor encephalitis. Ann Neurol. (2014) 76:108-19. doi: 10.1002/ana.24195

15. Peng X, Hughes EG, Moscato EH, Parsons TD, Dalmau J, Balice-Gordon RJ. Cellular plasticity induced by anti-alpha-amino-3-hydroxy-5-methyl4-isoxazolepropionic acid (AMPA) receptor encephalitis antibodies. Ann Neurol. (2015) 77:381-98. doi: 10.1002/ana.24293

16. Gleichman AJ, Spruce LA, Dalmau J, Seeholzer SH, Lynch DR. Anti-NMDA receptor encephalitis antibody binding is dependent on amino acid identity of a small region within the GluN1 amino terminal domain. J Neurosci. (2012) 32:11082-94. doi: 10.1523/JNEUROSCI.0064-12.2012

17. Mikasova L, De Rossi P, Bouchet D, Georges F, Rogemond V, Didelot A, et al. Disrupted surface cross-talk between NMDA and Ephrin-B2 receptors in antiNMDA encephalitis. Brain. (2012) 135:1606-21. doi: 10.1093/brain/aws092

18. Martinez-Hernandez E, Horvath J, Shiloh-Malawsky Y, Sangha N, MartinezLage M, Dalmau J. Analysis of complement and plasma cells in the brain of patients with anti-NMDAR encephalitis. Neurology. (2011) 77:589-93. doi: 10.1212/WNL.0b013e318228c136
Bochum, Germany for providing the NMDAR constructs used in this study, and Christina Burhoi, Institute for Genetics of Heart Diseases (IfGH), University of Muenster, Germany, for excellent technical assistance.
19. Bien CG, Vincent A, Barnett MH, Becker AJ, Blumcke I, Graus F, et al. Immunopathology of autoantibody-associated encephalitides: clues for pathogenesis. Brain. (2012) 135:1622-38. doi: 10.1093/brain/aws082

20. Planaguma J, Leypoldt F, Mannara F, Gutierrez-Cuesta J, Martin-Garcia E, Aguilar E, et al. Human N-methyl D-aspartate receptor antibodies alter memory and behaviour in mice. Brain. (2015) 138:94-109. doi: 10.1093/brain/awu310

21. Malviya M, Barman S, Golombeck KS, Planaguma J, Mannara F, Strutz Seebohm N, et al. NMDAR encephalitis: passive transfer from man to mouse by a recombinant antibody. Ann Clin Transl Neurol. (2017) 4:768-83. doi: $10.1002 / \mathrm{acn} 3.444$

22. Planaguma J, Haselmann H, Mannara F, Petit-Pedrol M, Grunewald B, Aguilar E, et al. Ephrin-B2 prevents N-methyl-D-aspartate receptor antibody effects on memory and neuroplasticity. Ann Neurol. (2016) 80:388-400. doi: 10.1002/ana. 24721

23. Seebohm G, Strutz-Seebohm N, Birkin R, Dell G, Bucci C, Spinosa MR, et al. Regulation of endocytic recycling of KCNQ1/KCNE1 potassium channels. Circ Res. (2007) 100:686-92. doi: 10.1161/01.RES.0000260250.83824.8f

24. Ferguson J, Mau AWH. Spontaneous and stimulated emission from dyes. Spectroscopy of the neutral molecules of acridine orange, proflavine, and rhodamine B. Aust J Chem. (1973) 26:1617-24. doi: 10.1071/CH97 31617

25. Kubin RF, Fletcher AN. Fluorescence quantum yields of some rhodamine dyes. J Lumin. (1982) 27:455-62. doi: 10.1016/0022-2313(82)90045-X

26. Moreau D, Lefort C, Burke R, Leveque P, O'connor RP. Rhodamine B as an optical thermometer in cells focally exposed to infrared laser light or nanosecond pulsed electric fields. Biomed Opt Express. (2015) 6:4105-17. doi: 10.1364/BOE.6.004105

27. Kreye J, Wenke NK, Chayka M, Leubner J, Murugan R, Maier N, et al. Human cerebrospinal fluid monoclonal N-methyl-D-aspartate receptor autoantibodies are sufficient for encephalitis pathogenesis. Brain. (2016) 139:2641-52. doi: 10.1093/brain/aww208

28. Castillo-Gomez E, Oliveira B, Tapken D, Bertrand S, Klein-Schmidt $\mathrm{C}$, Pan $\mathrm{H}$, et al. All naturally occurring autoantibodies against the NMDA receptor subunit NR1 have pathogenic potential irrespective of epitope and immunoglobulin class. Mol Psychiatry. (2017) 22:1776-84. doi: $10.1038 / \mathrm{mp} .2016 .125$

29. Levitan IB. It is calmodulin after all! Mediator of the calcium modulation of multiple ion channels. Neuron. (1999) 22:645-8. doi: 10.1016/S0896-6273(00)80722-9

30. Crisp SJ, Kullmann DM, Vincent A. Autoimmune synaptopathies. Nat Rev Neurosci. (2016) 17:103-17. doi: 10.1038/nrn.2015.27

31. Jezequel J, Johansson EM, Dupuis JP, Rogemond V, Grea H, Kellermayer $B$, et al. Dynamic disorganization of synaptic NMDA receptors triggered by autoantibodies from psychotic patients. Nat Commun. (2017) 8:1791. doi: 10.1038/s41467-017-01700-3

32. Hardingham GE, Bading H. Synaptic versus extrasynaptic NMDA receptor signalling: implications for neurodegenerative disorders. Nat Rev Neurosci. (2010) 11:682-96. doi: 10.1038/nrn2911

33. Melzer N, Biela A, Fahlke C. Glutamate modifies ion conduction and voltage-dependent gating of excitatory amino acid transporter-associated anion channels. J Biol Chem. (2003) 278:50112-9. doi: 10.1074/jbc.M 307990200

34. Melzer N, Torres-Salazar D, Fahlke C. A dynamic switch between inhibitory and excitatory currents in a neuronal glutamate transporter. Proc Natl Acad Sci USA. (2005) 102:19214-8. doi: 10.1073/pnas.0508837103

35. Manto M, Dalmau J, Didelot A, Rogemond V, Honnorat J. In vivo effects of antibodies from patients with anti-NMDA receptor encephalitis: further evidence of synaptic glutamatergic dysfunction. Orphanet J Rare Dis. (2010) 5:31. doi: $10.1186 / 1750-1172-5-31$ 
36. Seebohm G, Piccini I, Strutz-Seebohm N. Paving the way to understand autoantibody-mediated epilepsy on the molecular level. Front Neurol. (2015) 6:149. doi: 10.3389/fneur.2015.00149

37. Dey S, Temme L, Schreiber JA, Schepmann D, Frehland B, Lehmkuhl K, et al. Deconstruction - reconstruction approach to analyze the essential structural elements of tetrahydro-3-benzazepine-based antagonists of GluN2B subunit containing NMDA receptors. Eur J Med Chem. (2017) 138:552-64. doi: 10.1016/j.ejmech.2017.06.068

38. Gawaskar S, Temme L, Schreiber JA, Schepmann D, Bonifazi A, Robaa D, et al. Design, synthesis, pharmacological evaluation and docking studies of GluN2B-selective NMDA receptor antagonists with a Benzo[7]annulen-7-amine Scaffold. Chem Med Chem. (2017) 12:1212-22. doi: $10.1002 / \mathrm{cmdc} .201700311$
Conflict of Interest Statement: The authors declare that the research was conducted in the absence of any commercial or financial relationships that could be construed as a potential conflict of interest.

Copyright $\odot 2019$ Amedonu, Brenker, Barman, Schreiber, Becker, Peischard, StrutzSeebohm, Strippel, Dik, Hartung, Budde, Wiendl, Strünker, Wünsch, Goebels, Meuth, Seebohm and Melzer. This is an open-access article distributed under the terms of the Creative Commons Attribution License (CC BY). The use, distribution or reproduction in other forums is permitted, provided the original author(s) and the copyright owner(s) are credited and that the original publication in this journal is cited, in accordance with accepted academic practice. No use, distribution or reproduction is permitted which does not comply with these terms. 